\title{
Show Me! Guidelines for Producing Recorded Demonstrations
}

\author{
Catherine Plaisant ${ }^{1}$ and Ben Shneiderman ${ }^{123}$ \\ Human-Computer Interaction Laboratory, Institute for Advanced Computer Studies ${ }^{1}$, \\ Department of Computer Science ${ }^{2}$ \& Institute for Systems Research ${ }^{3}$ \\ University of Maryland, College Park, MD 20742 \\ \{plaisant,ben\}@cs.umd.edu
}

\begin{abstract}
Although recorded demonstrations (screen capture animations with narration) have become a popular form of instruction for user interfaces, little work has been done to describe guidelines for their design. Based on our experience in several projects, we offer a starting set of guidelines for the design of visually appealing and cognitively effective recorded demonstrations. Technical guidelines encourage users to keep file sizes small, strive for universal usability, and ensure user control etc. and provide tips to achieve those goals. Content guidelines include: create short demonstrations that focus on tasks, highlight each step with auditory and visual cues, synchronize narration and animation carefully, and create demonstrations with a clear beginning, middle, and end.
\end{abstract}

\section{Introduction}

Standardization and improved designs have made graphical user interfaces easier to learn and use, however getting started with unfamiliar interfaces, learning advanced features and understanding application domain concepts remains a challenge for many users [1], . As the goal of universal usability has become more prominent [2], especially in digital government applications, the need for dramatic improvements in tutorial and help methods has grown.

While government agencies seek to make their information resources and services available to an ever-growing set of users, guidelines for web design proliferate and improve by building on research results [3], but there is little research about guidelines for helping users get started with new web applications. Could effectively designed visual presentations be helpful?

Designers of any user interface documentation must make a variety of design decisions, including choosing an appropriate technology that matches the content. Paper documentation is valuable in presenting concepts, goals, and terminology, while graphics are helpful to convey site maps, state transitions, and data structures. Animations have long been promoted as an even more compelling way to educate users by showing the dynamics of user interface actions [4-8]. However, the research results have often surprised and frustrated the promoters of animations, since it is rare to find measurable benefits for users of animations [911]. User satisfaction with animations is usually high, but current animations appear to distract users from concentrating on key issues [12].

One theory is that animations lead mainly to mimicry and superficial learning [13]. Rieber, et al. [14] did a comparison study to examine the effects of different levels of visual elaborations (animated graphic, static graphic, and no graphic) on adult application learning in a computer-based science lesson. No significant results were found in this study when the static and animated visuals were added to the text. Palmiter et al. [11] compared the efficacy of learning computer-based tasks by using animated demonstrations, procedural textual instructions and combination of demonstrations and spoken text. They found that the demonstration groups were faster and more accurate for learning the procedural tasks, but, interestingly, seven days later the text group was faster and as accurate when performing identical and similar tasks. It seems that the retention issue needs to be addressed more fully. The results in Harrison's comparison study [15] suggested that using visual instruction in on-line help, either still graphic or animated, enabled the users to perform more tasks in less time and with fewer errors than those users who didn't have visual instruction, but there was no significant difference between the performance of subjects in the still graphic conditions and the animated conditions. 
A review of instructional uses of animation suggested that the effectiveness of animation appears to be confined to younger learners [16]. This review also reported that using animation to present content is less effective than other uses of animation, such as the four were identified by Rieber [14]: directing attention, presenting information, enhancing practice, and cosmetic appeal.

A more recent review covered research on computer animation in education, human-computer interaction and psychology [17]. These authors concluded that computer animation is not a panacea, but it can improve users' performance and attitude under certain circumstances. They summarized five factors that could influence the effectiveness of animation: the content to be animated, the level of interactivity, objective of animation, design of animated interface, and individual differences.

Rieber [18] presented three design recommendations for the use of animated visuals in instruction: (1) Animation should be incorporated only when its attributes are congruent to the learning task. Visualization, motion, and trajectory were three attributes considered to be brought by animation. (2) When learners are novices in the content area, they may not know how to attend to relevant cues or details provided by animation. (3) Animation's greatest contributions to computer based instruction (CBI) may lie in interactive graphic applications.

A study of students showed that the group that was given both animated and auditory descriptions about the operation of a bicycle tire pump performed better on the tests of creative problem solving than those only given animated description and those only given the auditory description [19].

Haas and al. [20] compared text, graphic and animation to provide meanings of statistical terms. There was little difference among formats in the effectiveness of presentations. User preferences varied but results suggest that the type of terms being explained made a difference, animations being more useful when there is an action or process. The lack of control of the timing of animations was seen as a problem.

These studies provided important guidance for our project to develop recorded demonstrations for webbased statistical software. We also studied the growing variety of animated demonstrations on the web to form and test our emerging hypotheses about guidelines for design. This paper presents a set of guidelines and tips to help designers prepare successful recorded demonstrations, which we playfully call "Show Me!" demonstrations. This term seems more active and conveys user control more strongly than the traditional term "Help", which has a desperate tone to it that may undermine user eagerness. We also reflect on the need and difficulty to quantify the relative benefit of design elements of recorded demonstrations, and summarize our experience and feedback from users.

\section{Exploration of recorded demonstrations}

A review of recorded demonstrations on the WWW that introduce users to a new interface revealed three major types:

Video movies show a person or the hands of a person using an interface. They are shot with a video camera and are effective at showing hardware manipulation or providing the nice touch of a human welcome. They are usually limited to a small window size for performance reasons. An example is the demonstration of body movement sensors of Ascension Technology (www.ascension-tech.com).

Composed animations include screen shots of the interface augmented with hand-made annotations (Fig. 1) and animations. A good example is available from Endeca (www.endeca.com) which provides an animation overview of the interface and an explanation of the search algorithm. The latter represents abstract concepts with graphical elements specially designed for the explanation and not from the interface itself. Those animations are usually created by multimedia professionals using tools such as Macromedia Director and Flash.

Recorded demonstrations demonstrate how a user interface can be used by replaying and narrating the recording of interface use. A good example can be seen at Amazon.com, which explains how to sell items (http://www.amazon.com/exec/obidos/subst/help/demo -sell-your-stuff/movie-window.html). Recorded demonstrations are simple to produce and narrate with recording tools such as Camtasia and generate faithful demonstrations of the interface.

Of course hybrid techniques exist as well and a recorded demonstration can be included in a composed animation. This paper focuses on the third type recorded demonstrations, because we believe they are a very effective method to provide help, and because they can be produced easily and therefore likely to be used widely. We also focus on the demonstrations of interfaces as opposed to explanations of concepts [20] 


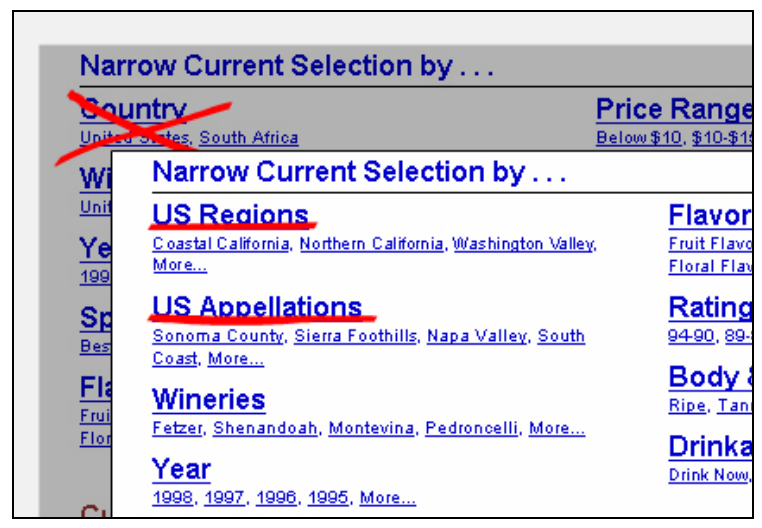

\section{Fig 1: Screen from a composed animation demonstrating the Endeca search. Cropped views of real screens are annotated and animated. (www.endeca.com/demos)}

Our guidelines are based on several threads of experience. First we have a long history of developing video demonstrations of our own work. Since 1991 the Human-Computer Information Laboratory has produced video reports at the occasion of its annual Symposium and Open House, and several segments have also become part of the $\mathrm{CHI}$ video proceedings. The videos served as archival record of our work and have been used by educators and researchers to illustrate interaction techniques to students and colleagues. Over the years we refined our techniques to write scripts and record short and effective videos in the space of a few hours with the help of a video professional pointing a camera at the screen.

More recently, we have worked on developing new methods for providing help to users of government websites offering statistical information. In particular we looked at how to help users get started with a tool allowing them to get information about US states using a map, scatterplots, table and sliders for filtering. This work led to a series of interfaces using 3 techniques: multi-layered design, a new help method called Integrated Initial Guidance, and recorded demonstrations [21, 22].

A usability test was conducted with 12 participants who used the first 2 techniques, then an additional test was conducted with 6 participants who learned the interface with the recorded demonstrations, and later were shown the other two techniques. Our observations suggested that the recorded demonstrations were quite effective, allowing users to get started quickly and complete the tasks. Most users seemed to have learned all aspects of the interface within the few minutes and were able to focus on the task immediately. Two seemed to learn the syntax of the application immediately (e.g. they learned how to move slider widget that they had never seen before) but seem to be unsure about the impact of the action on the display (moving a slider filtered out the display in a dynamic query manner) probably because of the faster pace and passive mode of demonstration. At least they were able to investigate on their own because they were intrigued by it and knew how to use the widgets. All users found the use of sound very important. They told us that after having used the methods with sound, other techniques appeared slow and less exciting. These early usability tests helped clarify guidelines such as the one in Section 3.5 to keep segments brief, use spoken narration, and be faithful to the interface. Other guidelines included practical suggestions such as keeping file sizes small.

In a follow-on project we generated 13 recorded demonstrations for our own tool, Treemap 4.0 (Fig. 2 and 3), which contributed to a substantial reduction in the number of questions received by email. These experiences led to further guidelines such as supporting universal usability.

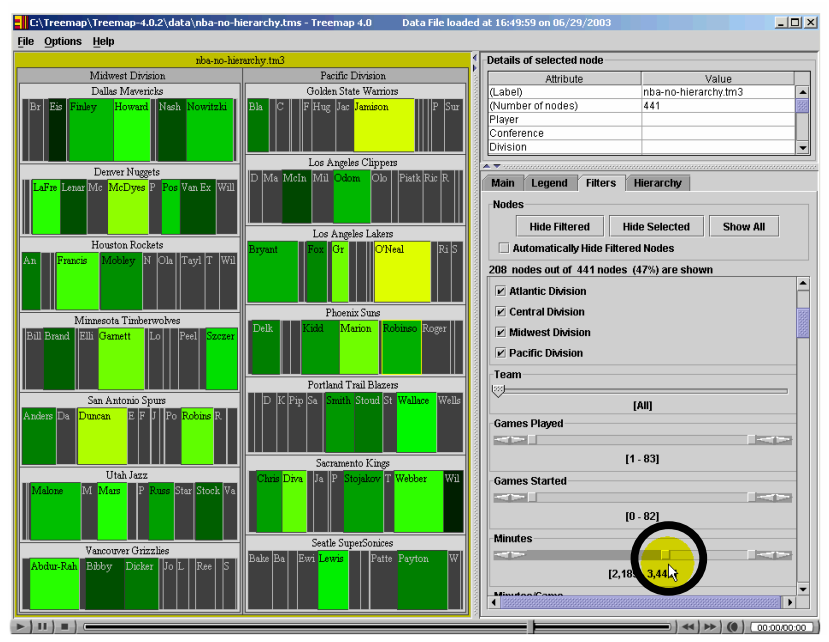

Fig. 2: A sample screen from a recorded demonstration of Treemap 4.0, produced with Camtasia. It shows the entire real screen of the application. Clicks can be heard, and the cursor is highlighted with a yellow circle. Here a slider in being moved. Synchronized voice commentaries explain the effect of this action on the display. (www.cs.umd.edu/hcil/treemap and visit the online documentation page) 

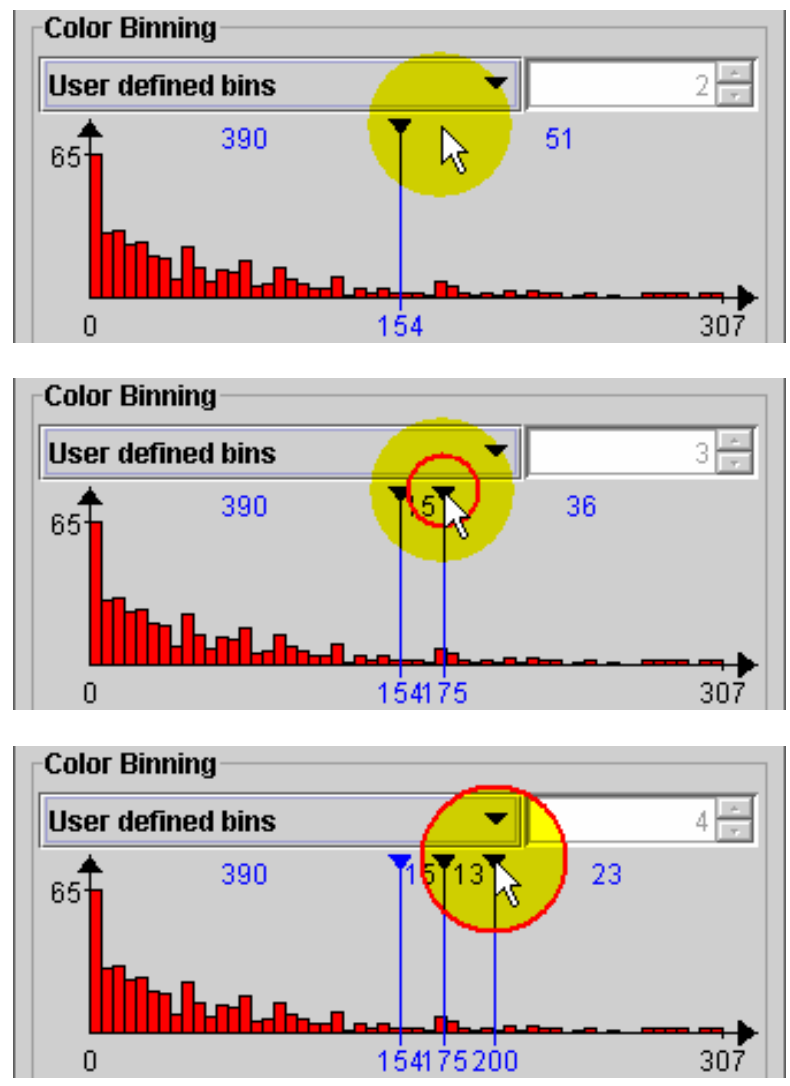

Fig. 3: Treemap 4.0 online documentation page offers this sequence that shows how to add bins to a histogram with a series of clicks (shown by red circles and a clicking sound).

To assess current practices we reviewed recorded demonstrations that we found on the web. While there were admirable examples, we also found many avoidable flaws. Therefore we prepared the guidelines in Section 3 to capture our experience and recommendations. Since one of our research projects focues on digital government, we emphasized US and state government sources (Appendix 1). This list is far from comprehensive and it focuses on usability aspects of these recorded demos:

Size: Size in kilobytes of the downloaded presentation

Buffering: Can the presentation start before it has finished downloading?

Length: Length in minutes and seconds

Pause: Can the user pause the presentation?

Seek: Can the user seek to a specific location in the presentation?

Time Display: Is there a control that indicates the position in the presentation?

Voice: Is there voice narration?
Text Boxes: Are there additional text boxes that are used in the presentation?

Click Cues: Are there audio/video cues to let the user know the mouse was clicked?

Highlighting: Was the mouse cursor or interface highlighted to help guide the user?

While our Treemap 4.0 recorded demonstrations were designed to have all these features, none of the others had the full set of features. Some of the recorded demos had excellent production values, such as professional narration and musical accompaniment, but the range was large. We found that one major improvement to content would be to remove long pauses and edit the demonstration more tightly. The recorded demonstrations without voice instructions were plainly less appealing and more difficult to follow, and smaller file sizes were a strong asset.

Finally we described our guidelines for recorded demonstrations to online help developers on many occasions and received enthusiastic approval of the rapid production technique, thereby encouraging us to recommend the technique and our guidelines more widely.

\section{Guidelines for recorded demonstrations}

We propose a set of ten guidelines and numerous tips to help designers create effective recorded demonstrations. Reviewing the guidelines in the context of the interface being demonstrated and the target user population, will lead to appropriate refinements. If access to these Show Me!'s is visible and attractive, but not intrusive, then users will feel in control and eager to try them.

\subsection{Provide procedural instruction rather than conceptual information}

Procedural instruction presents the steps required to complete a specific task, by walking users through the successful completion of the task. Conceptual information provides important theory and background information, but users may not need it to complete a task. Instructional information is a hybrid of procedural and conceptual information that encourages more comprehensive learning experiences for users. Keep the goal of showing procedure prominent in your thinking and scripting.

Tips:

- Base your script on a live demonstration (don't start from a paper)

- Pretend to be a user thinking-aloud while performing a real task 
- Do not advertise

\subsection{Keep segments short}

Short segments (15 to 60 seconds) keep users engaged while exploring the documentation and minimize how much users need to remember at once. Keeping each segment autonomous, while planning a linear sequencing between segments allows users to see them individually as needed, or all at once as a tutorial. Avoid showing multiple tasks in one segment. If you decide to combine tasks, consider using short titles to separate sections of the recording (example: "Printing", then later "Exporting"). Tips:

- Use the same example data or follow related examples across all segments.

- Starting the first segment with a tour of main screen components orients and introduces the vocabulary of the interface

- Starting each segment from a consistent screen also orients users.

\subsection{Ensure that tasks are clear and simple}

Choose understandable example tasks, and use familiar terms. Speak simply, directly, and accurately based on the following criteria; use active sentences, make positive assertions, keep sentence simple, speak directly to users, and state quantities exactly. Avoid abbreviations.

Tips:

- Prepare a script

- Use the same example data or follow related examples across all segments.

- Act out the interaction then describe changes seen on the screen

- Use concrete examples, e.g. do not say "selecting a color attribute with apply this attribute to all the objects presented on the screen". Instead say - and act out - "if you select "Gender" as the color attribute, all men are now colored blue and women red", and point at the effect as you talk.

- Edit, edit, edit, to cut all unnecessary words: e.g. do not say: "at the top left of the screen users will find the list of attributes, at the bottom right users can find the control panel". Instead say: "here is the list of attributes... and the control panel" while pointing or highlighting the corresponding areas, in synchronization with the narration.

\subsection{Coordinate demonstrations with textual documentation}

Often, recorded demonstrations complement textual documentation to provide users alternate ways of learning the materials. Textual documentation can provide more details and explain fundamental concepts that are not interactive. A good practice is to develop them in parallel so they use the same sequencing and terminology. Integrate textual documentation and demonstrations and let users choose what is most appropriate for them.

Tips:

- Make recorded demonstrations available right at the start of the application or where ever they are most likely to be needed by first-time users.

- Make the online documentation table of contents a table, clearly showing alternative formats available.

\subsection{Use spoken narration}

We highly recommend the use of voice narration (opposed to textual explanations). Users can easily attend to voice while watching the demonstration, and even possibly interacting with the interface. Narration keeps users engaged and paying attention. However, if narration and demonstration are not compatible or properly synchronized, it can confuse users. A common mistake is to have the voice give generic "advertisement-like" information about the interface while users are trying to understand what operation is actually being demonstrated on the screen. Another mistake is for the narration to be out-of-synch with the action.

Tips:

- Look for a narrator that has a lively voice and speaks clearly

- Favor a narrator who is likely to be around when time comes to update

- Ideally the same person will be able to control the interface and narrate at the same time to ensure good synchronization, but practically most people have difficulties doing this, and it often results in lengthy recordings. Our favored alternative is to work as a team, where one person uses the interface and the other narrates. Another alternative is to record demonstration and narration separately, then combine them with an editor. Several trials may be needed to adapt the narration speed and the speaker intonation to the rhythm of the interaction.

\subsection{Be faithful to the actual user interface}

Create a recorded demonstration that gives viewers the impression that they are watching the real interface. Showing the full size user interface helps users 
remember the location of interface widgets. Do not shrink the recorded demonstration if at all possible.

Tips:

- Zoom in on portions of the display when readability is a severe problem.

- If you cannot create a rapid and zoom transition to the detail area, first highlight the area where zooming will take place, then jump to it.

\subsection{Use highlighting to guide attention}

Narration can help understand what the user actions are but providing additional highlighting of the mouse cursor by adding circles, blinking features, or spotlights will help focus users' attention on where the action is. Sound effects can represent mouse clicks or sliding motion. There are many reasons to use sound in online documentation: to support visuals, to attract attention and interest, to improve perceptions of quality, to represent things not shown, to provide feedback, to aid memory, to preserve continuity, and so on. Likewise, in video documentation, sound plays an important role in attracting users' attention, providing feedback, and most importantly representing interactions between the interface and input device. Combining sound with the visual effect of an input device (mouse clicks or keyboarding sounds) often makes it easier for users to understand and recognize what is happening within the interface, and sometimes it allows representing more diverse input interactions. For example, combining a mouse click sound and an animated circle around the cursor help users easily recognize when and where the cursor is clicked over the interface. Moreover, changing the color of animated circle around the cursor makes it possible to distinguish left mouse click from right mouse click.

Tips:

- Don't use too many highlights, and avoid highlighting effects that can be confused with the interface itself.

- Wiggling the cursor is a simple way to attract attention and highlight a part of the screen, but stop moving the cursor afterward. Incessant and meaningless cursor movement will be dizzying, and confusing.

\subsection{Ensure user control}

If the recorded segment is longer that 15 seconds, give users controls so that they can skip the familiar parts, find the part they want or stop and replay. Speed control might be useful in some cases. Adding time length information and progress feedback helps users recognize their position in the recorded demonstration.

Providing jumps to the next segment in a logical sequence is also helpful.

\subsection{Keep file sizes small}

If the recorded demonstrations are designed to be displayed on the internet for online help, the response time has to be good since users will not have the patience to wait several minutes to see only a few seconds of demonstration. For example, suppose the recorded demonstration needs to be accessible through a $56 \mathrm{Kbps}$ dial-up modem connection, then the maximum size of a recorded demonstration that can be downloaded per minute is $420 \mathrm{~KB}(56 \mathrm{Kbps}$ (bit per second) $* 60$ seconds $=420 \mathrm{~KB}$ (bytes)). The benefits of rapid initiation are large, so streaming strategies are attractive.

Tips:

- Avoid traditional video recording, instead use onscreen recording of the interface which generates much smaller files and higher resolution.

- Experiment with different output formats, e.g. using Flash format might save a lot of space.

- Provide duration and file size for each segment

- Provide both streamed and downloadable versions.

\subsection{Strive for universal usability}

As mentioned in the previous guideline, the size of Show Me! files is an essential factor to make the recorded demonstrations practical for use in online documents. Nevertheless providing universal access will requires a careful review of technology choices. For example video codec, audio codec, plug-ins or dedicated players are often considered to reduce the size of files or shorten the response time. If designers can avoid these additional requirements, then the size of their user community will grow, especially among the large number of low-skilled computer users. Another consideration is to address the needs of users with disabilities. Providing transcripts of the narration for deaf users and textual descriptions for blind users will benefit others that have poor hearing or limited English competency. It also makes the demonstrations visible to search engines, and allow users to capture and print snapshots of the demonstrations. Control of the replay speed will be appreciated by everyone.

Tips:

- Indicate that your demo has audio

- Keep looking for new technology and formats that do not require users to install software. 


\section{Conclusions}

Instead of jumping to lengthy tutorials or manuals new users first intuition is to ask a colleague to give them a quick tour when they encounter a new interface. We believe that recorded demonstrations are a distant surrogate for such active and practical demonstrations. Although the benefits of recorded demonstration for learners of user interfaces have yet to be quantitatively measured, some investigations suggest that recorded demonstrations help users learn procedural tasks (see introduction). A set of controlled experiments could measure the benefits of individual features, but the diversity of interface and users will make an exhaustive evaluation challenging.

Our experience using Show Me!'s with our own products has been positive. For example the release of the recorded demonstration of Treemap has clearly changed the nature of the questions submitted to us. Before the release we would receive many questions about procedures e.g. "How do I change the colors?" or "Is there a way to do X" where $\mathrm{X}$ was possible and described in the text manual. Now we have NO questions of this nature, while questions about features that should be added, or licensing questions remain. We have received nice feedback from users such as:

"First of all, thanks very much for giving me the opportunity to explore your Treemap application with great ease. Through the self-explanatory demo, it allows me to truly appreciate the immense potential of such visual analytical application" A new treemap user from Singapore

We also observe a growing pattern of adoption (e.g. Amazon, Endeca) which suggests that web service providers find them effective, or at least easy enough to produce and keep up-to-date. A useful next step would be to gather usage data from those websites, e.g. are users of recorded demonstrations more likely to purchase? Another hypothesis is that users of recorded demonstrations are more likely to apply diverse features of an interface more effectively in their work.

\section{Acknowledgements}

We greatly appreciate the implementation efforts of Hyunmo Kang in developing our Treemap 4.0 recorded demonstrations. We also thank the students who helped us review current practices (Michael Anderson, John Booze, Pratik Mathur, Vikram Mittal and Dariush Samari) and all the members of the Govstat project (see http://ils.unc.edu/govstat/). This material is based upon work supported in part by the
National Science Foundation under Grant No. EIA 0129978, and the US Census Bureau.

\section{References}

[1] Carroll, J.M. (1997). Minimalism beyond the Nurnberg Funnel, MIT Press, Cambridge.

[2] Shneiderman, B. (2000). Universal Usability: Pushing human-computer interaction research to empower every citizen, Communications of the ACM 43, 5 (2000), 84-91.

[3] Koyani, Sanjay J., Bailey, Robert W., Nall, Janice R., and others (2003) Research-based Web Design E Usability Guidelines, Dept. of Health \& Human Services, National Institutes of Health Publication 03-5424, National Cancer Institute, Washington, DC. Available at http://www.usability.gov/pdfs/guidelines.html

[4] Baecker, Ronald, Small, Ian, and Mander, Richard (1991). Bringing icons to life, Proc. CHI '91 Human Factors in Computer Systems, ACM, New York, 1-6

[5] Payne, S. J., Chesworth, L., and Hill, E. (1992). Animated demonstrations for exploratory learners, Interacting with Computers, 4, 3-22.

[6] Wiedenbeck, S. and Zila, P. L. (1997). Hands-on practice in learning to use software: a comparison of exercise, exploration, and combined formats, $A C M$ Transactions on Computer-Human Interaction (TOCHI), 4(2), 169-196.

[7] Sukaviriya, Piyawadee "Noi" and Foley, James D. (1990). Coupling a UI framework with automatic generation of context-sensitive animated help, Proc. User Interface Software and Technology, ACM, New York, 152-166.

[8] Dominick, J., Hughes, A., Marchionini, G., Shearer, T., Su, Chang, and Zhang, J. (2003). Portal help: Helping people help themselves through animated demos, Univ. of North Carolina Information \& Library School Technical Report http://ils.unc.edu/ils/research/reports/TR-2003-01.pdf

[9] Morrison, Julie B., Tversky, Barbara, and Betrancourt, M. (2000). Animation: Does it facilitate learning, Proc. Workshop on Smart Graphics, AAAI Press, Menlo Park, CA.

[10] Morrison, J. B. and Tversky, B. (2001). The (in)effectiveness of animation in instruction, Proc. CHI 2001 Human Factors in Computer Systems: Extended Abstracts, ACM, New York, 377-378.

[11] Palmiter, S., Elkerton, J. and Baggett, P. (1991). Animated demonstrations vs. written instructions for learning procedural tasks: A preliminary investigation, International Journal of Man-Machine Studies, 34, 687-701.

[12] Weiss, Renee E., Knowlton, Dave S., and Morrison, Gary R. (2002). Principles for using animation in computer based instruction: Theoretical heuristics for effective design, 
Computers in Human Behavior 18, 465-477.

[13] Atlas, R., Cornett, L., Lane, D. M., \& Napier, H. A. (1997) The use of animation in Software Training: Pitfalls and Benefits. In M. Quinoñes and A. Dutta (Eds.) Training for 21st Century Tachnology: Applications of Psychological Research. American Psychological Soc., Washington, D.C

[14] Rieber, L. P. (1994). Computers, Graphics and Learning, Brown \& Benchmark Publishers, Madison, WI

[15] Harrison, S.M. (1995). A comparison of still, animated, or non illustrated on-line help with written or spoken instructions in a graphical user interface, Proceeding of $A C M$ CHI'95 Conference on Human Factors in Computing Systems, ACM, New York, 82-89

[16] Wetzel, C. D., Radtke, P. H., \& Stern, H. W. (1994) Instructional effectiveness of video media. Hillsdale, NJ: Erlbaum

[17] Bétrancourt, M. and Tversky, B. (2000). Effect of computer animation on users' performance: a review. Le travail Humain, 63(4), 311-330.
[18] Rieber, L. P. (1991). Animation, incidental learning, and continuing motivation. Journal of Educational Psychology, 83(3), 318-328.

[19] Mayer, E., and Anderson, R. B. (1991). Animations need narrations: An experimental test of a dual-coding hypothesis, Journal of Educational Psychology 83, 484-490

[20] Haas, S., Brown, R., Cao, L., and Wilbur, J. (2005). Evaluation of the GovStat Statistical Interactive Glossary: Implications for Just-in-Time Help. U. of North Carolina Technical Report, http://ils.unc.edu/govstat

[21] Plaisant, C., Kang, H., and Shneiderman, B. (2003). Helping users get started with visual interfaces: multi-layered interfaces, integrated initial guidance and video demonstrations, Proc. Human-Computer Interaction International 2003: Vol. 4 Universal Access in HCI, Lawrence Erlbaum Ass., Mahwah, NJ (2003), 790-794.

[22] Kang, H., Plaisant, C., and Shneiderman, B. (2003). New approaches to help users get started with visual interfaces: Multi-layered interfaces and integrated initial guidance, Proc. 2003 National Conf. on Digital Government Research (2003), http://www.dgrc.org/dgo2003/.

\section{Appendix 1:}

A sample of recorded demonstration examples, collected during our exploration of current practices. We collected features related to the usability aspects of recorded demos from state government web sites and other sources.

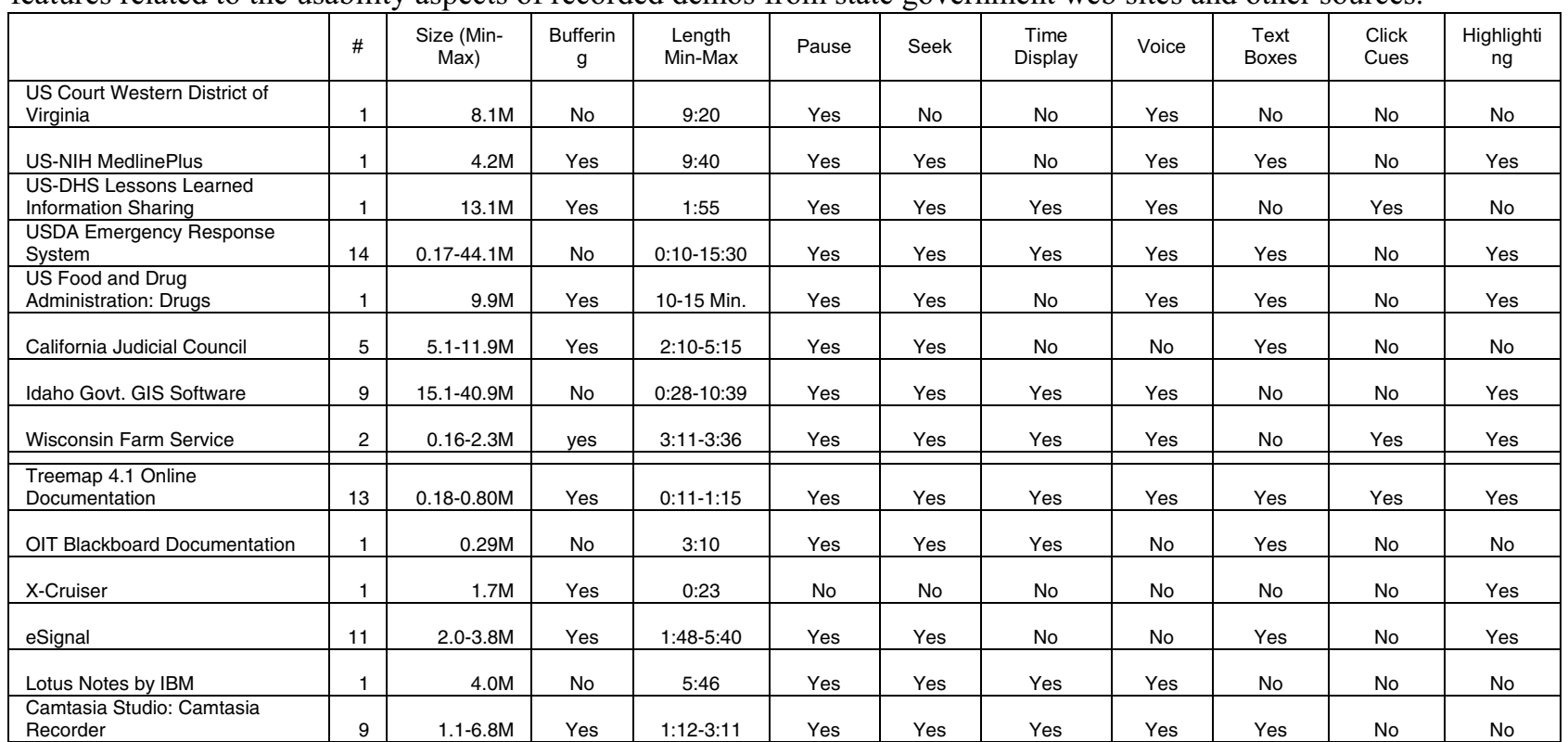

